Original Article

\title{
The effect of different visual stimuli on reaction times: a performance comparison of young and middle-aged people
}

\author{
Makoto Otaki, OTR, MS ${ }^{1,2)^{*}, \text { Katsuyuki Shibata, OTR, PhD }}{ }^{3)}$ \\ 1) Faculty of Rehabilitation, Kobegakuin University, Japan \\ 2) Division of Health Science, Graduate School of Medical Science, Kanazawa University: \\ 518 Arise Ikawadani-cho, Nishi-ku, Kobe, Hyogo 651-2180, Japan \\ 3) Faculty of Health Science, Institute of Medical, Pharmaceutical and Health Sciences, \\ Kanazawa University, Japan
}

\begin{abstract}
Purpose] Here, we evaluated the reaction times of young and middle-aged people in different tasks. [Participants and Methods] The study included 23 young and 28 middle-aged volunteers. Their reaction times were measured in three tasks featuring different symbols (arrow and figure symbols) and spatial attributes (left, right, and ipsilateral choices). [Results] No significant inter-group differences in the reaction times were found for the simple reaction time task. In the choice reaction time and go/no-go reaction time tasks, the middle-aged participants demonstrated significantly slower reaction times. When the correct response was congruous with the direction of an arrow stimulus, the reaction times were shortened significantly among the middle-aged participants. In the go/ no-go reaction time task, the reactions were delayed due to an inhibition of responses to upcoming stimuli. [Conclusion] The slower reaction time of the middle-aged participants in the choice reaction time task suggested that their responses were guided by the arrow stimulus to a greater extent compared to that of the younger participants. In the go/no-go reaction time task, the reaction times may have been slower in middle-aged participants because of a non-response possibility, which meant that participants had to first check the stimulus before deciding whether to respond.

Key words: Middle-aged, Reaction time, Response characteristics
\end{abstract}

(This article was submitted Oct. 26, 2018, and was accepted Dec. 19, 2018)

\section{INTRODUCTION}

When driving a vehicle, we make split-second decisions based on instantaneous visual information. To perform appropriate actions in everyday life, we sometimes focus more on a particular stimulus, or we might delay our response to a stimulus to check whether the response is appropriate. According to Lewis et al., older people have diminished neuromotor function ${ }^{1)}$. Reaction time has been used to measure age-related response quality ${ }^{2}$. There are three types of reaction time tasks: simple reaction time (simple RT), choice reaction time (choice RT), and go/no-go reaction time (go/no-go RT) ${ }^{3}$. MacDonald et al. found that older age groups exhibited longer reaction times and greater intra-individual variability in reaction time ${ }^{4}$. Many studies have compared the reaction times of young and old individuals ${ }^{5)}$, but little is known about the reaction times of middle-aged people. Rehabilitative interventions cover a broad age range, so it is essential that such interventions are tailored to the response characteristics corresponding to the person's age.

This study aimed to ascertain the characteristics of young and middle-aged people's reaction times in different reaction time tasks from multiple perspectives. The ultimate goal was to provide findings that can guide rehabilitative interventions.

*Corresponding author. Makoto Otaki (E-mail: ohtaki@reha.kobegakuin.ac.jp)

(C2019 The Society of Physical Therapy Science. Published by IPEC Inc.

(c) (1) $\odot$ This is an open-access article distributed under the terms of the Creative Commons Attribution Non-Commercial No Derivatives cc) ${ }_{\text {BY NC ND }}$ (by-nc-nd) License. (CC-BY-NC-ND 4.0: https://creativecommons.org/licenses/by-nc-nd/4.0/) 
To this end, we conducted two experiments. The first consisted of simple RT and choice RT tasks; the second consisted of a go/no-go RT task in which responses were inhibited.

\section{PARTICIPANTS AND METHODS}

We recruited 51 healthy volunteers, 23 of whom were young ( 9 males and 14 females; average age: $20.5 \pm 0.6)$ and 28 of whom were middle-aged (16 men and 12 women; average age: $47.7 \pm 8.0$ ). We confirmed that each participant was righthanded using the Edinburgh Handedness Inventory ${ }^{6}$. Each of these individuals provided their informed consent to participate after receiving a briefing about the study's purpose. The study was approved by Kobe Gakuin University's ethics committee for human experimentation (No. HEB 17-15).

In the experiments, we used a personal computer, E-Prime 2.0, and Chronos (Psychology Software Tools, Inc.). When performing the tasks, the participants sat approximately $80 \mathrm{~cm}$ from the PC screen with their fingers poised on the response buttons (Fig. 1 left). The participants were presented with a fixation point for 3,000 ms. After this, the target stimulus appeared and the participants had to execute a response (Fig. 1 right). Each task consisted of a brief instruction, 10 practice rounds, and then 20 rounds in which we measured reaction times.

We only analyzed the reaction times of correct responses. We excluded reaction times of $<100 \mathrm{~ms}$ deemed as "too early" and reaction times of $>2,000 \mathrm{~ms}$ deemed as "too late." The stimuli were randomized for each participant.

The statistical software we used was SPSS (version 24). We performed a multiple comparison (Bonferroni correction) on the three choice variables in the choice RT and go/no-go RT tasks (left/right, ipsilateral, contralateral). We used a paired t-test to compare reaction times between the two symbolic variables (arrow symbol, figure symbol) in the choice RT task. We used an unpaired t-test to compare the reaction times of the young and middle-aged participants. We set the statistical significance threshold at $>5 \%$. For the path analysis, we used SPSS Amos (version 24).

In the simple RT task, participants had to press a response button as quickly as possible when a stimulus appeared in the middle of the screen. In the choice RT task, each stimulus corresponded to one of three choice variables: left and right choice (LR choice), ipsilateral choice (IL choice), or contralateral choice (CL choice). For LR choice, the stimulus appeared in the middle of the screen and participants had to press either the left or right-hand button. For IL choice, participants had to press the left-hand button if the stimulus appeared on the left of the screen, and the right-hand button if it appeared on the right. For CL choice, participants had to press the right-hand button if the stimulus appeared on the left of the screen and vice-versa. The target stimuli consisted of two types of symbols: 1. an arrow (arrow symbol) that pointed in the direction of the button to press; and 2. a dot or cross (figure symbol) that did point in a direction. The arrow and figure symbols appeared at a ratio of 1:1, as did the two responses (left and right). The go/no-go RT task included the same three choice variables as the choice RT task - LR choice, IL choice, and CL choice. However, it also included a no-response condition ("no-go"). Two types of stimuli were used in this task: a black dot, which indicated that the response should be executed ("go"); and a white circle, which indicated that no response should be executed ("no-go"). The black dot and white circle appeared at a ratio of 1:1. The ipsilateral and contralateral choices appeared at a ratio of $3: 2$.

\section{RESULTS}

Table 1 shows the reaction times for the simple RT and choice RT tasks. In each task, around 1\% of the responses were erroneous. None of the responses were too early or too late. To start with the young people, when the stimulus was an arrow, reaction times for the CL choice were significantly slower compared to that for the LR and IL choices ( $<<0.01$ ). With a figure
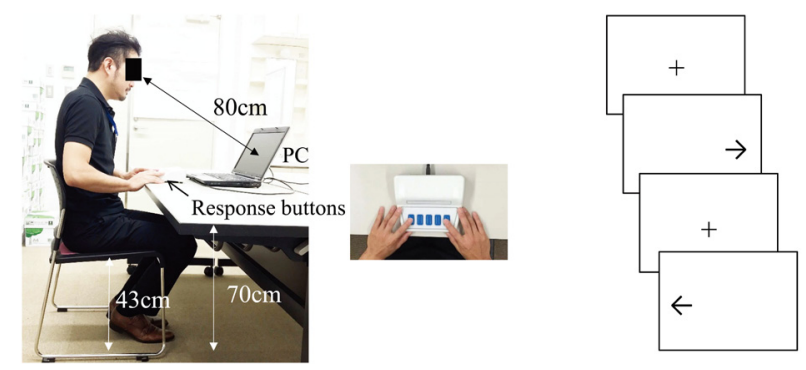

Fig. 1. Experimental setting with an overhead view of response buttons (left), and visual stimulus samples (right).

The left figure shows the experimental setting, including the position of the desk, chair, and screen, with an overhead enlarged view of the response buttons. The right figure is a sample of the visual stimulus in the choice reaction task, indicates the direction of the passage of time. $\leftarrow$ : Arrow symbol, $\bullet$ : Figure symbol. 
Table 1. Reaction time (ms)

\begin{tabular}{|c|c|c|c|c|c|c|c|c|c|}
\hline \multicolumn{2}{|c|}{ Task $\cdot$ symbol } & Response & Young & $\begin{array}{c}\text { Choice } \\
\text { comparison }\end{array}$ & $\begin{array}{l}\text { Symbolic } \\
\text { comparison }\end{array}$ & Middle & $\begin{array}{c}\text { Choice } \\
\text { comparison }\end{array}$ & $\begin{array}{l}\text { Symbolic } \\
\text { comparison }\end{array}$ & $\begin{array}{c}\text { Age } \\
\text { comparison }\end{array}$ \\
\hline \multicolumn{2}{|l|}{ Simple } & & $232 \pm 41$ & & & $228 \pm 41$ & & & \\
\hline \multirow[t]{6}{*}{ Choice } & Arrow & LR choice & $314 \pm 52$ & & & $336 \pm 53$ & & & $* *$ \\
\hline & & IL choice & $315 \pm 64$ & $\mathrm{~b} \dagger \dagger$ & & $344 \pm 61$ & $\mathrm{~b}+\dagger$ & & $* *$ \\
\hline & & CL choice & $415 \pm 92$ & $\mathrm{c} \dagger$ & & $439 \pm 96$ & $\mathrm{ct \dagger}$ & & $* *$ \\
\hline & Figure & LR choice & $351 \pm 69$ & $\mathrm{a} \dagger \dagger$ & $d \dagger \dagger$ & $372 \pm 85$ & $\mathrm{a} \dagger \dagger$ & $d \dagger \dagger$ & $* *$ \\
\hline & & IL choice & $309 \pm 53$ & $\mathrm{~b}+\dagger$ & & $336 \pm 60$ & $\mathrm{bi \dagger}$ & & $* *$ \\
\hline & & CL choice & $391 \pm 110$ & $\mathrm{ct \dagger}$ & $\mathrm{e} \dagger$ & $409 \pm 84$ & $\mathrm{c \dagger \dagger}$ & $\mathrm{e} \dagger$ & $*$ \\
\hline \multirow{3}{*}{\multicolumn{2}{|c|}{ Go/no-go }} & LR choice & $333 \pm 57$ & $\mathrm{a} \dagger \dagger$ & & $361 \pm 64$ & $\mathrm{a} \mathrm{a}^{\dagger}$ & & $* *$ \\
\hline & & IL choice & $371 \pm 74$ & $\mathrm{~b}+\dagger$ & & $415 \pm 79$ & $\mathrm{~b}+\dagger$ & & $* *$ \\
\hline & & CL choice & $452 \pm 122$ & $\mathrm{c \dagger \dagger}$ & & $498 \pm 98$ & $\mathrm{ct \dagger}$ & & $* *$ \\
\hline
\end{tabular}

$\mathrm{n}=51$ (for each task).

All data are presented as the mean \pm SD. Simple: Simple RT task; Choice: Choice RT task; Go/no-go: Go/no-go RT task; Arrow: Arrow symbol; Figure: Figure symbol; Response: Spatial attribute of button to press; Choice comparison: Comparison of left/right ipsilateral, and contralateral choice variables; Symbolic comparison: Comparison of the two symbol variables; Age comparison: Comparison of young and middle-aged participant responses; LR choice: Left and right choice; IL choice: Ipshilateral choice; CL choice: Contralateral choice. a: Left/right vs. Ipsilateral, b: Ipsilateral vs. Contralateral, c: Left/right vs. Contralateral, d: Left/right with arrow symbol vs. Left/right with figure symbol, e: Contralateral with arrow symbol vs. Contralateral with figure symbol.

$\dagger \mathrm{p}<0.01, * \mathrm{p}<0.05, * * \mathrm{p}<0.01$.

symbol, reaction times were slowest for the CL choice followed by the LR and IL choices. Reaction times were quicker with an arrow than with a figure symbol. Of the choice variables, the LR choice was associated with the quickest reaction times, and the CL choice with the slowest.

As for the middle-aged participants, their reaction times were similar to the young participants overall. Although their reaction times in the simple RT task were not significantly different from that of the young participants, those in choice RT were significantly slower in the choice task in all conditions.

Table 1 also shows the reaction times for the go/no-go RT task. Among the young participants, reaction times were quickest for the LR choice $(\mathrm{p}<0.01)$ followed by the IL and CL choices. We observed a similar pattern in the middle-aged participants' reaction times, but these reaction times were significantly slower than those of the young participants in all conditions.

To determine the difficulty level of each task variant, we compared the responses in all tasks on a Brinley plot ${ }^{7)}$. The results revealed a linear relationship with an incline of 1.15, indicating that the reaction time of the middle-aged group increased as the difficulty level rose in each task. The most difficult task variants were go/no-go RT task with CL choice, arrow symbol with CL choice, and figure symbol with CL choice. We performed a covariance structure analysis (maximum likelihood estimation) to model the relationships of the task variants. The analysis of all measured variables yielded facilitating and inhibiting variables $\left(\chi^{2}=33.6, p<0.01\right.$, RMSEA $=0.023$; Fig. 2$)$. We extracted the variables based on the standardized coefficients. There was one facilitating variable: arrow symbol with LR choice. There were two inhibiting variables: 1 . figure symbol with CL choice, and 2. go/no-go RT task.

\section{DISCUSSION}

Age did not appear to influence reaction times in the simple RT task. In the choice RT and go/no-go RT tasks, however, reaction times were slower among middle-aged participants, who had more response choices associated with slower reaction times. Since response choices were more easily guided by an arrow symbol indicating a direction, more time was needed to determine selection of responses to left and right and whether to press the response button in tasks involving figure symbols.

Compared to figure symbols, the arrow symbol was associated with significantly quicker reaction times. According to affordance theory, instantaneous visual information elicits specific actions immediately without the mediation of complex cognitions or decisions ${ }^{8)}$. Arrows are stimuli that contain information known by the perceiver such that attention is attracted in the direction of the arrow. As such, arrows can facilitate choice responses, which explains why the path analysis revealed the arrow symbol to be a facilitating factor for reaction times. Reaction times were significantly quicker in the IL choice than they were in the CL choice. DeJong et al. describes the response processes as follows; after the stimulus is presented, the stimulus is identified, the response is selected, and then the response is executed ${ }^{9}$. . Responses are selected for stimuli during the response-selection step ${ }^{10}$. In the case of the IL choice, the response and stimulus are congruous (both are on the same side), so the response is selected automatically. In the CL choice, however, the response and stimulus are incongruous (they are on opposite sides), so the automatically selected response must first be inhibited, and then the response opposite to the automatic response must be selected. Therefore, in the CL choice, stimulus identification conflicts with stimulus response ${ }^{11)}$. These procedural differences in response selection explain why the IL choice was associated with quicker reactions times 


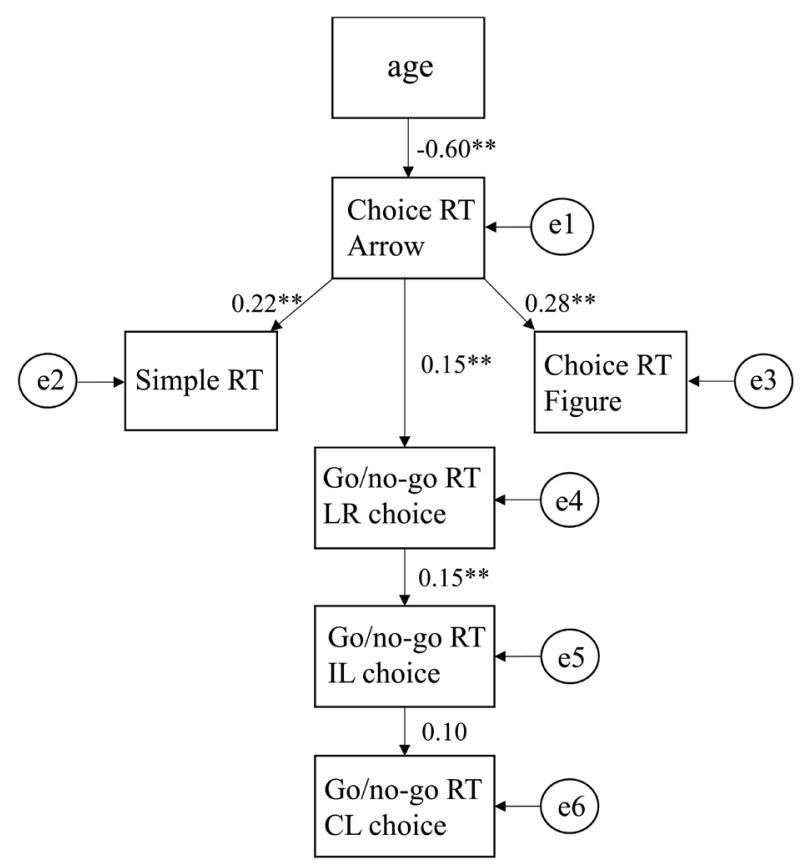

Fig. 2. Path analysis of facilitating and inhibiting factors.

The figures shown on the paths indicate standardized coefficients. Simple RT: Simple reaction time; Choice RT: Choice reaction time; Go/no-go RT: Go/no-go reaction time; Arrow: Arrow symbol; Figure: Figure symbol; LR choice: Left and Right choice; IL choice: Ipsilateral choice; CL choice: Contralateral choice. $*$ * $<<0.01$

while the CL choice were associated with slower reaction times. At higher difficulty levels, the go/no-go RT task was associated with slower reaction times than the choice RT task. In the choice RT task, every stimulus required a response. In the go/ no-go RT task, however, only a "go" stimulus required a response ("no-go" required a non-response). According to Miller et al., a "go" decision activates a prepared response, but when "no-go" is a possibility, this prepared response must be inhibited until the person has checked whether it should be executed ${ }^{12)}$. Accordingly, the go/no-go RT task increased the impetus to check the response against the stimulus. The presence of this checking process would explain why reaction times were slower in this task, as well as why the path analysis indicated go/no-go RT task as an inhibiting variable.

Reaction times were slower in general among both young and middle-aged participants; we noted an age effect. Given the tendency for an arrow symbol to elicit a response toward the direction it is facing, the presence of this symbol as the stimulus may have generated conflict between the spatial attribute of stimulus side and that of the response, resulting in delayed reaction times. The go/no-go RT task was more difficult because of the possibility of a no-go condition. The presence of the no-go condition may have delayed reaction times because the stimuli needed to be checked (as to whether it is "go" or "no-go") before the response could be executed.

We only analyzed correct responses. Future studies should additionally analyze incorrect responses, including "go" responses to "no-go" stimuli. There is a trade-off between response time and response accuracy, and we did not control for this relationship in our study. Additionally, male may generally respond faster than females; such a gender bias shall be considered in the future studies which need to examine the effect in a more gender-balanced population.

\section{Conflicts of interest}

We have no conflicts of interest to declare.

\section{ACKNOWLEDGEMENT}

We wish to thank all the students and faculty staff in the Faculty of Rehabilitation, Kobegakuin University who cooperated as participants. 


\section{REFERENCES}

1) Lewis RD, Brown JM: Influence of muscle activation dynamics on reaction time in the elderly. Eur J Appl Physiol Occup Physiol, 1994, 69: 344-349. [Medline] [CrossRef]

2) Salthouse TA: The processing-speed theory of adult age differences in cognition. Psychol Rev, 1996, 103: 403-428. [Medline] [CrossRef]

3) Donders FC: On the speed of mental processes. Acta Psychol (Amst), 1969, 30: 412-431. [Medline] [CrossRef]

4) MacDonald SW, Nyberg L, Sandblom J, et al.: Increased response-time variability is associated with reduced inferior parietal activation during episodic recognition in aging. J Cogn Neurosci, 2008, 20: 779-786. [Medline] [CrossRef]

5) Spirduso WW: Reaction and movement time as a function of age and physical activity level. J Gerontol, 1975, 30: 435-440. [Medline] [CrossRef]

6) Oldfield RC: The assessment and analysis of handedness: the Edinburgh inventory. Neuropsychologia, 1971, 9: 97-113. [Medline] [CrossRef]

7) Ratcliff R, Spieler D, McKoon G: Explicitly modeling the effects of aging on response time. Psychon Bull Rev, 2000, 7: 1-25. [Medline] [CrossRef]

8) Gibson JJ: The ecological approach to visual perception. Boston: Houghton Mifflin, 1979.

9) De Jong R, Liang CC, Lauber E: Conditional and unconditional automaticity: a dual-process model of effects of spatial stimulus-response correspondence. J Exp Psychol Hum Percept Perform, 1994, 20: 731-750. [Medline] [CrossRef]

10) Hommel B, Prinz W: Theoretical issues in stimulus-response compatibility. Amsterdam: North-Holland, 1997.

11) Hasbroucq T, Guiard Y: Stimulus-response compatibility and the Simon effect: toward a conceptual clarification. J Exp Psychol Hum Percept Perform, 1991, 17: 246-266. [Medline] [CrossRef]

12) Miller J, Schäffer R, Hackley SA: Effects of preliminary information in a Go versus No-go task. Acta Psychol (Amst), 1991, 76: 241-292. [Medline] [CrossRef] 